Andrews University

Digital Commons @ Andrews University

Faculty Publications

$12-1-2004$

\title{
Failure in Internally Pressurized Bent Tubes
}

\author{
Robin Stevenson \\ General Motors \\ Boon-Chai $\mathrm{Ng}$ \\ Andrews University,ngb@andrews.edu \\ Peter Polidoro \\ Seagull Solutions Inc.
}

Follow this and additional works at: https://digitalcommons.andrews.edu/pubs

Part of the Materials Science and Engineering Commons

\section{Recommended Citation}

Stevenson, Robin; Ng, Boon-Chai; and Polidoro, Peter, "Failure in Internally Pressurized Bent Tubes" (2004). Faculty Publications. 2156.

https://digitalcommons.andrews.edu/pubs/2156

This Article is brought to you for free and open access by Digital Commons @ Andrews University. It has been accepted for inclusion in Faculty Publications by an authorized administrator of Digital Commons @ Andrews University. For more information, please contact repository@andrews.edu. 


\title{
Failure in Internally Pressurized Bent Tubes
}

\author{
ROBIN STEVENSON, BOON-CHAI NG, and PETER POLIDORO
}

\begin{abstract}
The analysis and modeling of tube-hydroformed components is more complicated than that employed for sheet-metal panels, due to the lengthier process sequence and variable strain path-from flat-rolled sheet to tube; from straight tube to bent tube; and from bent tube to hydroformed component. These additional process steps make it difficult to determine whether post mortem analyses of tube failure during hydroforming can, and should, be conducted with the same tools and databases as used for simple stampings. To provide a partial answer, the properties of commercially fabricated welded straight tubes were evaluated using a free-expansion internal pressure test and compared with those of free-expansion internal pressure tests on bent tubes. The results demonstrated that the behavior of the bent tube was consistent with the mechanical properties of the as-received tube, provided due notice was accorded to the complex strain history of the bent tube. However, due to the strain-path changes occurring at the failure location, conventional approaches for monitoring strain history would yield (apparently) anomalous results.
\end{abstract}

\section{INTRODUCTION}

TUBE hydroforming using seamless welded tube has become a major process for fabricating automotive structural components. The process is deceptively simple: a tube is bent to the approximate shape of the desired component, placed in a die of the appropriate shape, and internally pressurized (typically, using a water-based fluid) so that it deforms to fill the die cavity and adopts the desired shape.

However, a multiplicity of deformation events are experienced by the material of the tube wall. ${ }^{[1]}$ Even before the hydroforming process itself, the tube is fabricated by deforming flat-rolled product by bending it into an "O" shape, welding the edges together, and then further deforming it to develop the desired size and ovality. Thus, the tube-wall properties differ from those of the flat sheet from which it was fabricated and also vary through the thickness.

The tube is then bent (typically, locally) to conform to the die geometry, thereby introducing strain gradients both along the tube (corresponding to the bent and unbent regions) and "across" the tube (corresponding to the inner and outer bend radii).

Finally, the tube is expanded by internal pressurization and is subjected to a strain state largely corresponding to plane-strain expansion. Typically, varying amounts of expansion are imposed along the length of the tube, since one of the production advantages of tube hydroforming is its ability to generate varying cross sections along the tube.

Thus, any element of the tube may have been subjected to a series of deformations of varying strain paths during the overall fabrication process. ${ }^{[1]}$

There is continuing interest in modeling this process and in performing post mortem analyses of failed processes to understand whether these failures are more related to material

ROBIN STEVENSON, Senior Staff Research Engineer, is with General Motors Research and Development Center, Warren, MI 48090-9055. Contact e-mail: Robin.Stevenson@gm.com BOON-CHAI NG, Associate Professor, is with the Engineering Technology Department, Andrews University, Berrien Springs, MI 49104. PETER POLIDORO, Staff Engineer, is with Seagull Solutions Inc., Morgan Hill, CA 95037.

Manuscript submitted November 1, 2002. characteristics or process complexity. One element of any modeling or analysis process is to relate the material failure to its forming-limit curve. Unfortunately, it is difficult to accurately determine the mechanical properties of the tube wall due to its geometry, which confounds attempts to perform simple tensile or dome tests. A more natural approach would be to evaluate the properties of the original sheet prior to tube fabrication and to track their evolution as the process proceeds.

However, although the recently developed stress-based approaches ${ }^{[2]}$ hold promise, the well-known complexity of material behaviors under varying load paths ${ }^{[3,4]}$ largely frustrates this approach. Hence, attempts to trace the material behavior back to the initial sheet properties or even to the tube properties and, particularly, its forming-limit behavior, have encountered difficulties. ${ }^{[5]}$

Thus, the objective of this study was to extend some earlier studies on the expansion of straight tubes to address the expansion of bent tubes.

\section{EXPERIMENTAL}

All testing was conducted on (nominal) 69.85-mm (2.75-inch) o.d., 2-mm wall thickness, continuous-welded mild steel tubing.

These tubes, which were approximately $3 \mathrm{~m}$ in length, were bent around a radius of $139.7 \mathrm{~mm}$ (5.5 inches or 2 times diameter) to $90 \mathrm{deg}$ at two locations using a rotary draw-bending process (for details, refer to Reference 6 ) on a computer numerically controlled tube bender, using an internal mandrel to prevent tube collapse. The weld region represents a local zone in which both the tube microstructure and properties deviate from the bulk of the tube. It was, therefore, considered desirable to explicitly investigate the influence of the weld on processing. This was done by locating the bend axis at two distinct positions relative to the weld: the first configuration located the weld on the nominal neutral axis, and the second configuration located the weld on the inner (compression) side of the bend, where the strain is maximum. In subsequent discussion, these two configurations will be referred to as the weld-onneutral-axis (WN) and the weld-in-compression (WC) tubes, respectively. 
After bending, the tubes were cut into two bent "elbows," with each elbow incorporating two straight sections approximately $150 \mathrm{~mm}$ in length for mounting the elbow into the pressurization fixture. Prior to pressurization, the geometry of the tubes was verified using a coordinate measuring machine equipped with a Moiré interferometry head. By projecting a pattern of alternating parallel light and dark bars on the tube and recording and analyzing their image on the tube, it is possible to determine the geometry of the illuminated patch. By analyzing multiple patches and combining the information from all the patches, a three-dimensional picture of the tube can be developed.

The pressurization fixture is shown in Figure 1. It comprises two clamps, bolted to a die plate, which securely position and locate the tube. In preliminary experiments, it was observed that the tube tended to expand and burst on the straight sections used to mount the tube rather than in the bent section (Section IV). To suppress this behavior, the tube elbows were cut to minimize the length of straight tube, and two steel collars were clamped on the exposed straight section of the tube to force deformation in the bent section only.

Pressurized fluid is introduced into the tube, and sealing is achieved with a fixture comprising a doughnut shaped piece of polyurethane sandwiched between two steel discs. The entire assembly is supported on a hollow threaded rod which serves as the conduit for introduction of the pressurized fluid. After this is inserted into the tube, the polyurethane doughnut is expanded radially by driving the steel discs together by tightening a nut down the threaded rod. By matching the polyurethane doughnut to the tube inner diameter, the expanding polyurethane contacts the tube wall, deforms to conform to local irregularities, and effects a seal. The entire assembly was located on the bed of a 200-ton press, and ram pressure was applied to the clamps for added restraint.

Pressure was applied using a hydraulic pump/intensifier unit capable of developing pressures of up to $135 \mathrm{MPa}$. Pressure was continually monitored during the test using a pressure sensor mounted on the outlet line of the pump/intensifier and was recorded on a personal computer (PC)-based data-acquisition unit running National Instru-

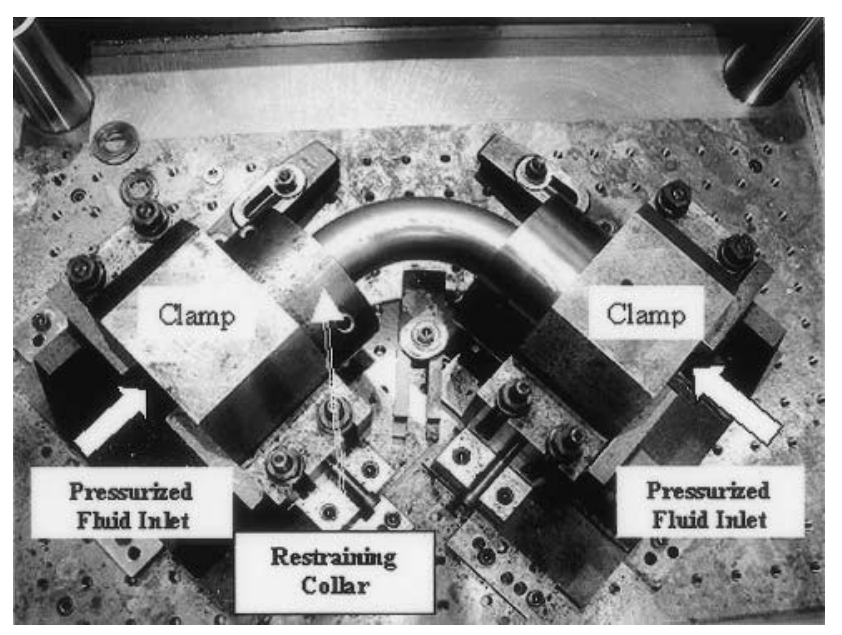

Fig. 1-Overview of burst test apparatus. ments LOOKOUT* ${ }^{*}$ software. Pressurization was termi-

*LOOKOUT is a trademark of National Instruments, Austin, TX.

nated when tube rupture occurred.

Two approaches were followed to measure strain. Electroetched $2.54-\mathrm{mm}$ circle grids were applied to the asreceived tubes, and then the circles were measured using a commercial camera-based strain-measurement system* after

*Available from Hitchcock-Manthey LLC, Troy, NY.

bending and testing, to make post facto assessments of the deformation occurring during these processes. In addition, strain gages were applied to specific locations on selected bent tubes prior to expansion, permitting an on-going measurement of the local strain history experienced by these locations during pressurization. The strain-gage data were also recorded on the PC-based data-acquisition system.

It should be noted that the circle-grid measurements are subject to a number of possible errors associated with the difficulty of using the electroetching process on a tube and of reading the circles on a curved surface. In addition, the electroetching process, chosen because the circles are more resistant to being rubbed off during contact with the bending die, typically generates more ragged circles than those generated by the photoresist process and, thus, causes, the camera system more problems in determining their dimensions. To minimize the impact of these problems, nominal circle dimensions were not used as the basis for strain measurements. Instead, circles, randomly chosen on the surface of the undeformed tube, were read prior to bending, and these dimensions were used for strain assessments. Despite these precautions, it would be optimistic to assume that the accuracy of the circle-grid strain data exceeds \pm 3 pct.

Work hardening in the tube wall was investigated using hardness measurements made at a series of locations at the midwall thickness on polished tube cross sections cut from the center of the bend transverse to the tube longitudinal direction. Hardness indentations were made using a Rockwell "N" brale under $15 \mathrm{~kg}$ major load. To ensure stability under loading, the tube section was mounted on an oversized piece of sheet metal using epoxy. The requirement that the indentation be at least 2.5 times the indentation diameter from a free surface and any other indentation was satisfied, even at the smallest tube-wall thickness.

\section{RESULTS}

Representations of the tube cross sections after bending, inferred from the three-dimensional coordinate measuring machine data at six locations, is shown at approximately $50 \%$ of life size in Figure 2(b) and, at higher magnification in Figure 2(c). The positions of these six locations relative to the bend is shown in Figure 2(a). It may be noted that two of these six measurements were made in the straight sections where no bending occurred. These cross sections, thus, act as internal references of the initial tube geometry against which the sections measured in the bent section may be compared. Clearly, as indicated in Figure 2(b) and in greater detail in Figure 2(c), no pronounced distortion of the tube geometry occurred during bending, and the initially circular cross section 
is maintained. The data indicate that the maximum deviation recorded at any point from one section to another is less than $1 \mathrm{~mm}$.

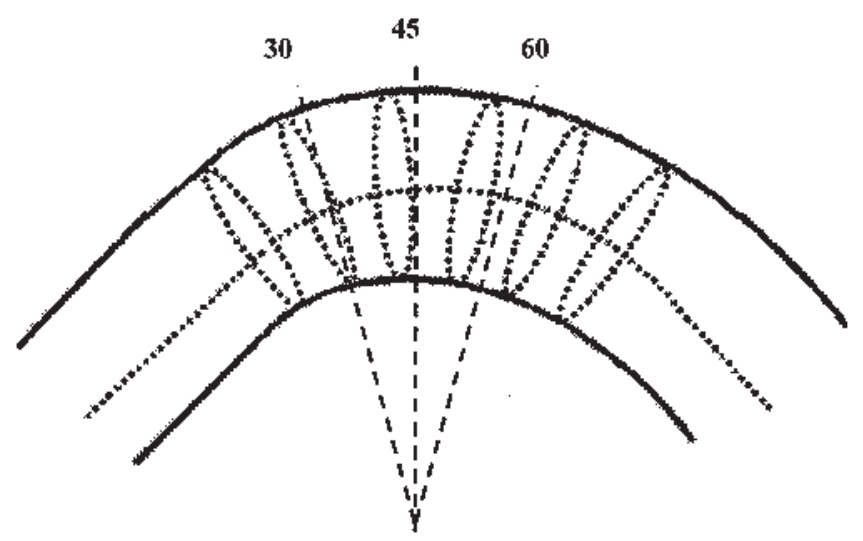

(a)

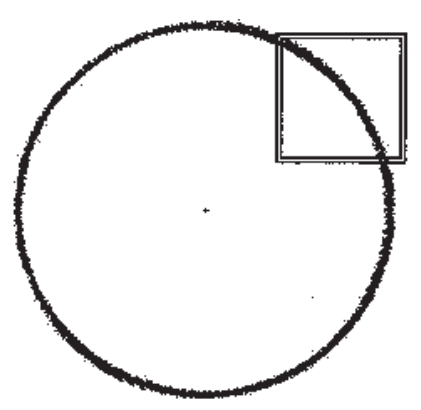

(b)

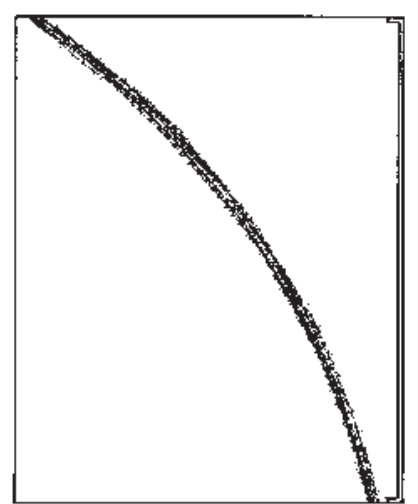

(c)
Fig. 2-(a) Sketch illustrating the locations of the sections cut in the bent tube and the overlay of these sections at (approximately) (b) 50 pct and (c) 175 pct of full size. Sketch (a) also illustrates the sections for the strain measurements shown in Fig. 3.
Circle grid-derived strains measured around the circumference of the bend at three locations (detailed in Figure 2(a)) are shown in Figure 3. Three features are immediately apparent. First, the weld has minimal influence on the bending strain since, within experimental error, both the WC and WN tubes show similar strains. Second, the bend is not a pure bend, since the sum of the maximum strains recorded on the opposing sides is nonzero and indicates that approximately 5 pct tensile deformation is superimposed on the bending deformation, displacing the neutral axis off the tube centerline toward the compression side of the bend. (Similar behavior may be observed in Figure 3 of Reference 6, but, surprisingly, not in Figure 16 of Reference 6). Third, the sample-to-sample variation is remarkably small; similar consistency was observed in thickness measurements made using an ultrasonic thickness gage.

Figures 4 and 5 compare the strain distribution measured around the tube after pressurization to failure in the vicinity of the fracture, with the strain distribution measured prior to pressurization but after bending. It is clear that the strain distributions (again, making due allowance for measurement uncertainty) differ only at the neutral-axis locations. For the WN tube, one of these locations approximates the weld location, but similar behavior is observed for the WC tube, where the weld is well removed from the neutral axis, confirming that the weld has minimal influence on the fracture location. Thus, it is apparent not only that the failure site seeks out the neutral axis, but also that the deformation is extremely localized, with locations even $3 \mathrm{~mm}$ away from the fracture site showing no evidence of additional deformation over and above that resulting from bending.

This observation is reinforced by the results obtained on bursting the strain-gaged tubes. Figures 6 and 7 show the locations of strain gages placed on a WN tube and the strains recorded by each gage. It is clear that significantly greater strains were recorded by gage 1 (closest to the fracture) than by any of the other gages. Also, the maximum strain recorded on gage 1 is significantly less than the strains measured from circle-grid measurements, even though the gage length of

Circumference Measurement: $M N$ and WC Tubes

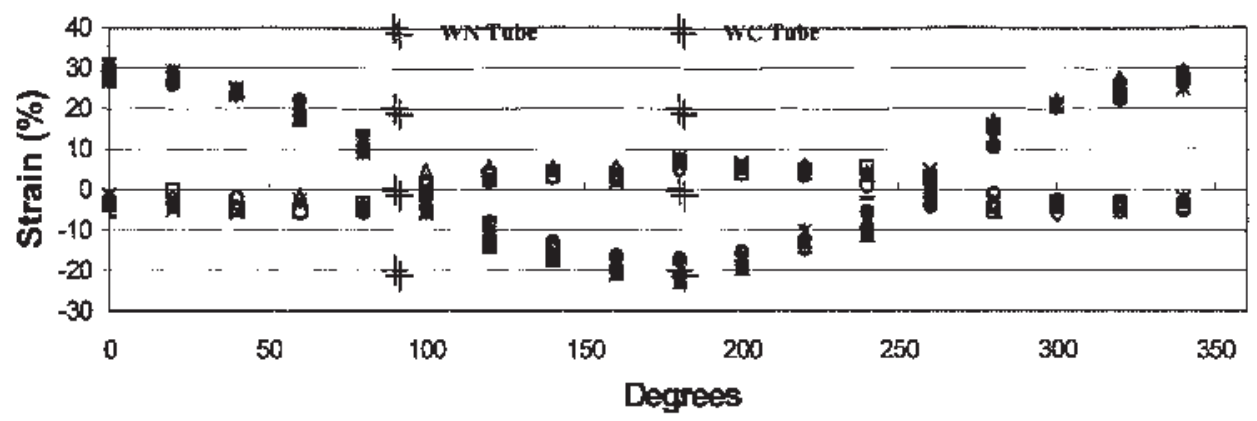

+WN 30 Axial Strain
+WN 60 Aval Strain
-WE 45 Audal Strain
+WEO

- WN 30 Ciranterential Stain AWN 45 Axial Strain.

CWN 60 Cramterential Strain e Mo 30 Axial Strain

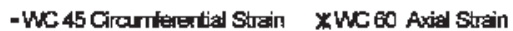

$\triangle$ ¿WN 45 Cinanferential Strain

OWC 30 Crumterential Strain

XMc 6oGiomfential strain

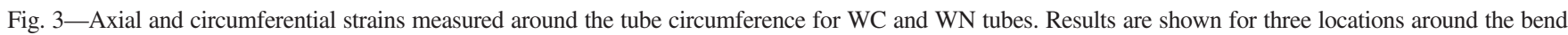

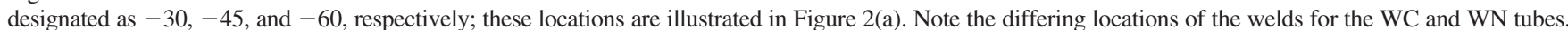




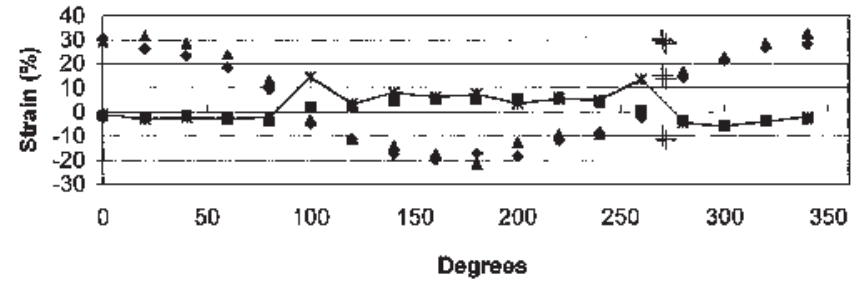

\begin{tabular}{ll}
\hline Before Burst Axial Strain & - Before Burst Circumferential Strain \\
* After Burst Axial Strain & *-After Burst Circumfenential Strain \\
+ Weld &
\end{tabular}

Fig. 4-Comparison of axial and circumferential strains after bending and after bending and burst testing for WN tube.

Comparison of strains before and after burst test: WC Tube

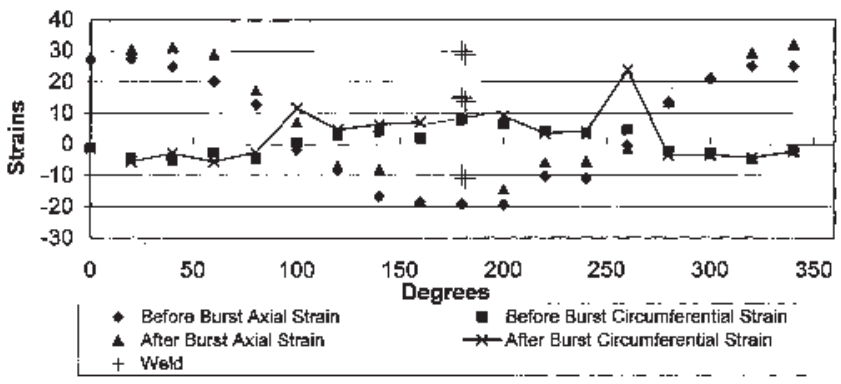

Fig. 5-Comparison of axial and circumferential strains after bending and after bending and burst testing for WC tube.

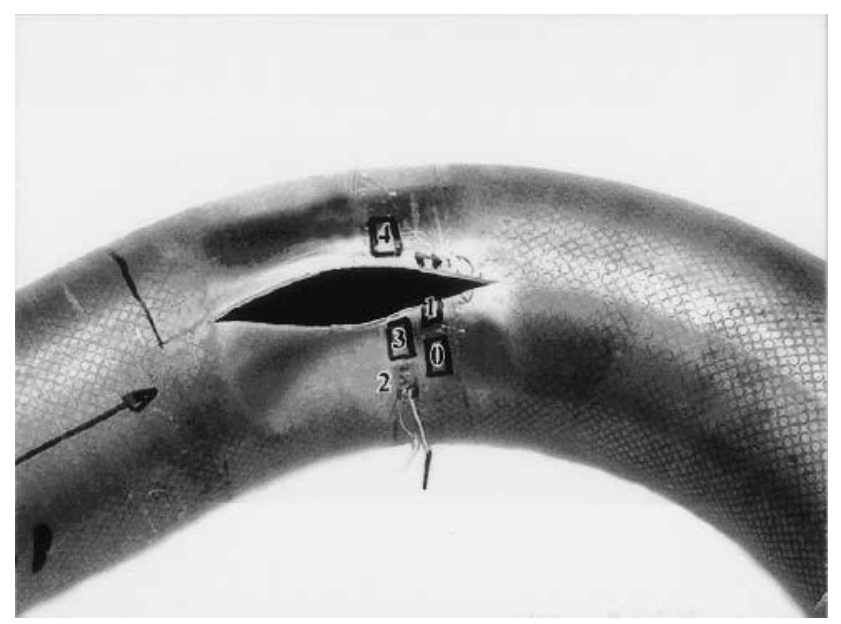

Fig. 6-Photograph of burst WN tube after failure illustrating strain gage locations.

the gage $(3.175 \mathrm{~mm})$ is comparable to the circle dimension. However, strain-gage decohesion occurred prior to tube failure for gage 1, although not for the other, less-highly strained gages. Thus, while the low-strain results observed on gages 2 , through 4 (no signal was recorded for gage 0 ) may be considered reliable, the higher terminal strain recorded by gage 1 underestimates the strain at that location.

Similar observations were made on a WC tube, as shown in Figures 8 and 9. In this case, the results are more dramatic,

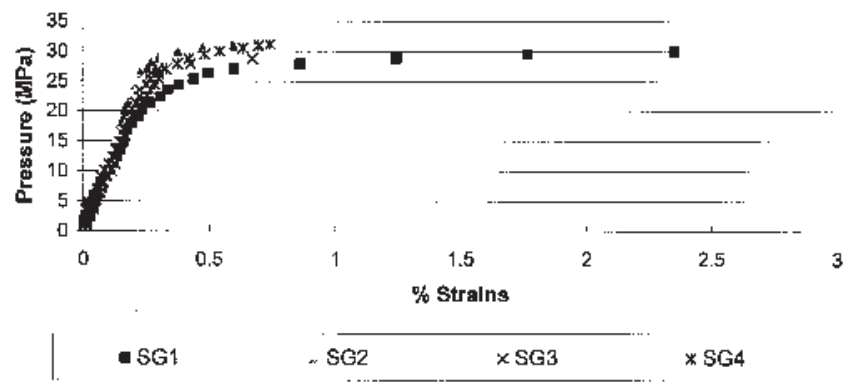

Fig. 7—Pressure-strain record for tube and strain gages shown in Fig. 6.

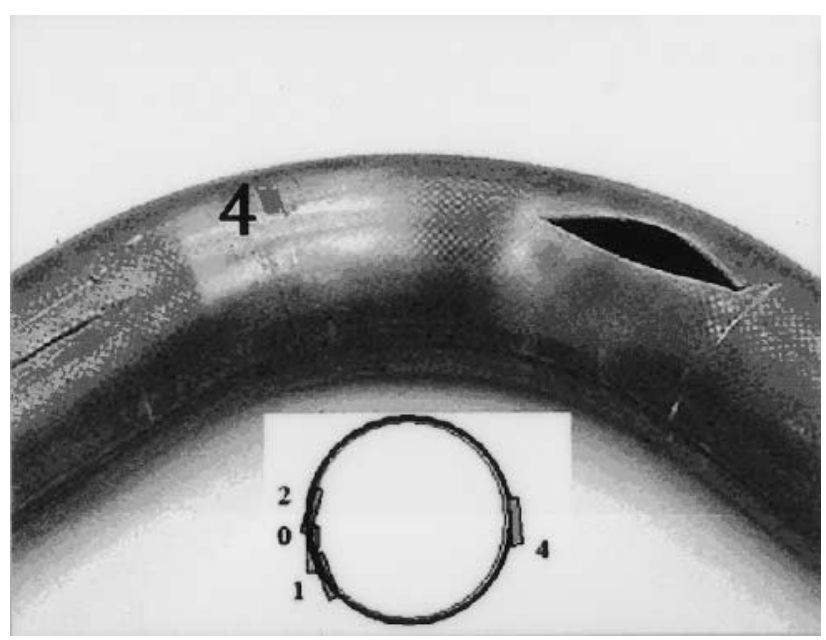

Fig. 8-Photograph of burst WC tube and inset illustrating strain gage locations. Note that gage 4 is located just above the failure line on the same side of the tube (plan view) as the fracture but on the opposite side of the apex of the curve, while gages 0,1 , and 2 are on the opposing side.

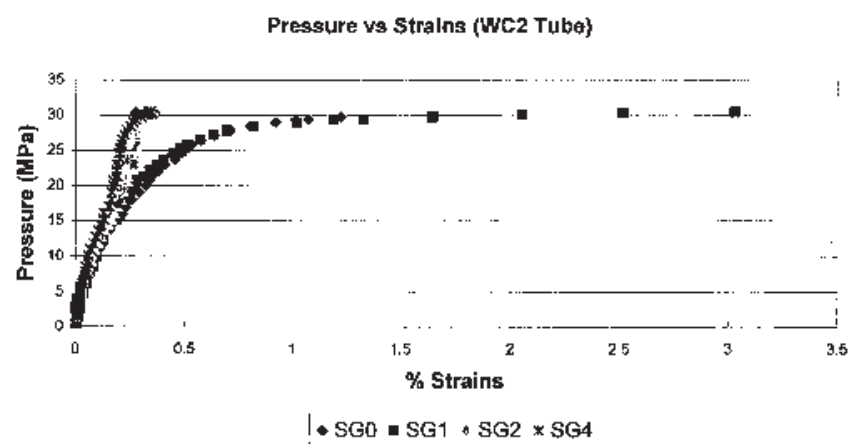

Fig. 9-Pressure-strain record for tube and strain gages shown in Fig. 8.

with the two strain gages furthest from the neutral axis exhibiting essentially elastic behavior, while only the two strain gages closest to the neutral axis exhibit a plastic response. Again, this indicates that the observed behavior tracks with the bending of the tube and not with the weld.

Under conditions of pure bend, it is clear that there must exist a region of null strain, i.e., the neutral axis, and that this must be the wall region with the lowest flow stress. It 
is also obvious that a bend and superimposed tensile strain must exhibit an axis of null macroscopic strain (neutral axis). However, it is not clear that this must necessarily corresponds to the wall region with the lowest flow stress, since the null macroscopic strain corresponds to a compressive bending strain offset by a tensile strain of equal magnitude. Depending on the magnitude of the Bauschinger effect, this means that the "neutral axis," far from being strain-free, has been deformed to a strain of up to twice the tensile strain. Thus, the measured, postbending, strain-free zone does not necessarily correspond to the zone of lowest flow stress.

However, as shown by Figure 10, which provides companion plots of the (Rockwell 15N) hardness and (the absolute value of) thickness strain around the tube perimeter of a WC tube, the zero-strain location (neutral axis) does correspond to the lowest hardness- R15N 55. (A parallel series of hardness tests was conducted for a WN tube, and very similar results were obtained. Thus, these results reflect the influence of only the bend axis and not the weld.) A study ${ }^{[9]}$ that explicitly investigated the relationship between hardness and flow behavior demonstrated a correlation between yield stress and (Vickers) hardness. Assuming similar behavior in this case, these results demonstrate that the neutral axis is the site at which the wall flow stress is a minimum.

Note that samples of as-received straight tubes showed a range of hardness of $\mathrm{R} 15 \mathrm{~N} 45$ to 51 . In keeping with the results of Wang et al. ${ }^{[9]}$ this variation, although complicated by "noise" resulting from experimental variability, showed a strong systematic variation with angular position and is considered to reflect the local deformation experienced by the initially flat sheet as it is fabricated into a tubular shape. Recollecting that the minimum hardness in the bent tube was $\mathrm{R} 15 \mathrm{~N} 55$, it is clear that the bending operation has introduced significant work hardening into all regions of the tube, including the neutral axis. Clearly, this is consistent with the overall imposed tensile strain indicated by the axialstrain data.

In contrast to the work of Wang et al.,${ }^{[9]}$ no numerical relationship between hardness and yield strength was sought in this study; thus, it is impossible to assign specific strength levels to these hardness values.

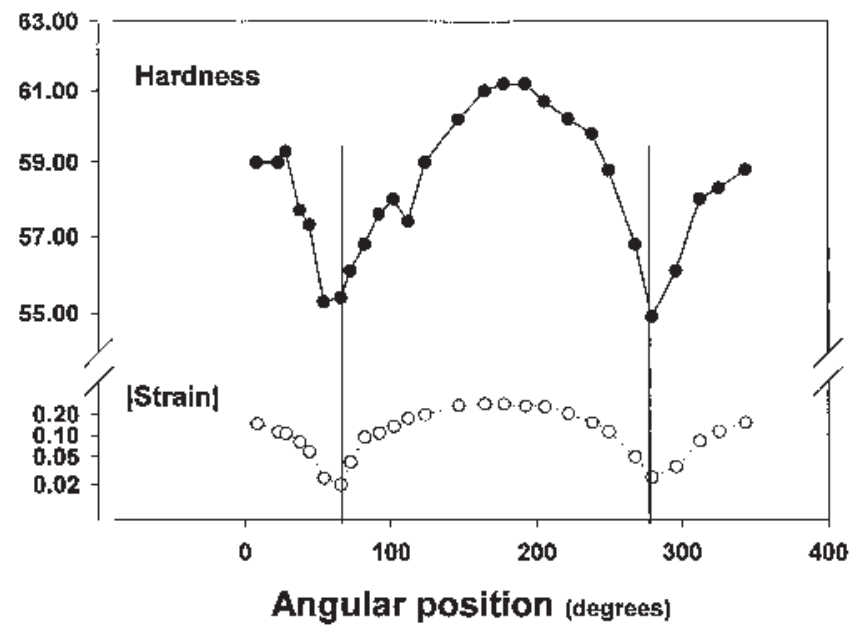

Fig. 10-Overlaid plots of hardness (R15N) and thickness strain around the wall of a bent tube. Zero degrees corresponds to the weld location.
However, the applied stress in the strain-gaged bent tube wall can be compared to the stress-strain curve-generated data taken during the expansion of a similarly strain-gaged straight tube. Anisotropic behavior originating either from the intrinsic tube-wall material properties or from local inhomogeneities associated with the tube-making process was ignored in the analysis.

Since the strain gages indicated that the strain state in the middle of the tube approximated plane strain, the instantaneous wall thickness and tube diameter for the straight tube could be derived from the strain measurements recorded by the strain gages, and the usual relations* could be employed

$$
\begin{aligned}
& * \varepsilon_{\text {Effective }}=\frac{\sqrt{2}}{3} \sqrt{\left(\varepsilon_{1}-\varepsilon_{2}\right)^{2}+\left(\varepsilon_{2}-\varepsilon_{3}\right)^{2}+\left(\varepsilon_{3}-\varepsilon_{1}\right)^{2}} \text { and } \\
& \sigma_{\text {Effective }}=\frac{1}{\sqrt{2}} \sqrt{\left(\sigma_{1}-\sigma_{2}\right)^{2}+\left(\sigma_{2}-\sigma_{3}\right)^{2}+\left(\sigma_{3}-\sigma_{1}\right)^{2}}
\end{aligned}
$$

to derive the effective stress and strain.

This result was then compared with the flow stresses derived from the bent-tube pressure-strain data. Again, stress determinations were based on the measured pressures and tube geometries, and the tube was modeled as both straight and as a section of a torus (for details, refer to the Appendix) tube geometry.

The bent tube was analyzed using an approach similar to that used for the straight tube, despite its nonuniform wall thickness and wall strength. Since the strain data are taken directly from the deforming region, however, the wall thickness in the deforming region will be accurately tracked. Given the very localized deformation observed and the relatively small strain experienced prior to failure, the instantaneous tube diameter was assumed to be equal to the initial tube diameter. This should not be a major source of error, since the total strain recorded by the strain gages was $\sim 3$ pct, indicating that changes in the tube diameter and, hence, possible errors in the computed stress would be of similar magnitude.

Note that both approaches to analyzing the bent-tube analyses are approximations, even under conditions where the bend has a constant wall thickness and uniform wall properties. The straight-tube analysis ignores the in-plane tube curvature, while the toroidal analysis is suspect due to the local stresses at the transition between the bent (quarter-torus) and straight sections of the sample.

The stress-strain curve, derived from the pressure-strain data, for the straight, as-received tubes is shown in Figure 11. In addition a stress-strain curve from the bent tubes is also shown, with its origin located at strains of 0 and 0.10 . The bent-tube data located at an origin of 0 (zero) strain are shown analyzed both as a straight tube and as a torus.

However, as is clear by comparing Eqs. [A1] and [1], the differences between these analyses are minor and, realistically, indistinguishable. Thus, for clarity, at a strain of 0.10 , only the straight-tube analysis is shown.

The justification for replotting the data displaced by a strain of 0.1 is the observation that the tube experiences a tensile strain of 5 pct in addition to the bending strain. Since the circle grids at the location of interest show no evidence of deformation after bending is complete, the region must have experienced a bending compressive strain of 0.05 , which negated the imposed 0.05 tensile strain. However, assuming that the Bauschinger effect is minimal, under this strain history, the 


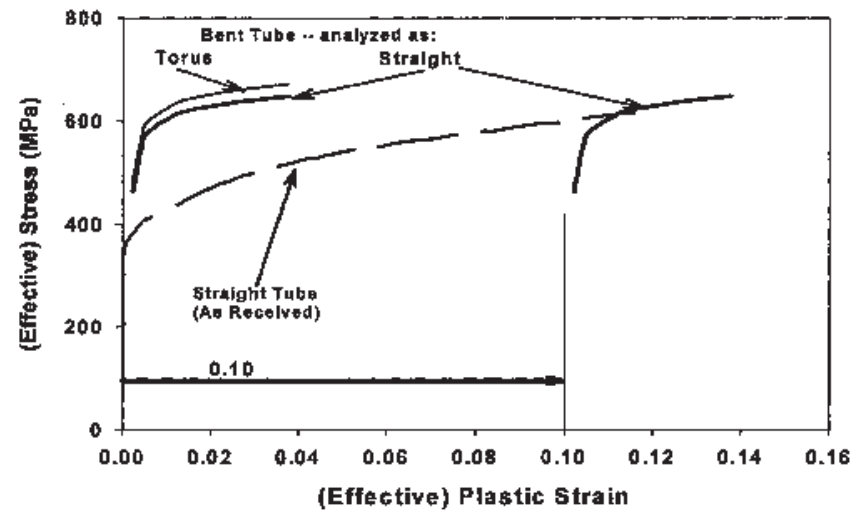

Fig. 11-Comparison of stress-strain curves for straight and bent tubes. The bent tube results are presented based on an andysis assuming a straight tube and an analysis assuming a torus. The bent tube results are shown with an origin at zero strain-corresponding to a direct analysis of the pressure-strain data-and also with an origin located at a strain of 0.10 to indicate the range of (bending) prestrains experienced at the failure location prior to pressurization.

material will work harden as though it had experienced a cumulative strain of $0.10(=0.05+|-0.05|)$.

When the data are displaced by 0.10 strain, it is clear that the data from the bent and as-received tubes overlay one another. Thus, the implication of this result is that the severe strain localization observed results from the diminution of the work-hardening capability of the tube resulting from the bending operation. Given the simplifications of the analysis, more extensive interpretation is not merited. (Recollect that anisotropy in the tube mechanical properties was not considered; the determination of 5 pct tensile elongation during bending was based on the circle-grid measurements, whose accuracy was assessed as $\pm 3 \mathrm{pct}$; and the imposed tensile deformation during tube bending was imposed in the axial direction, while the strain resulting from internal pressure is circumferential.)

It is worth noting that, irrespective of the near-identical stresses computed using the toroidal and straight-tube analyses, the philosophical question of whether the straight or toroidal analyses "best" describe the deformation and rupture behavior is also clouded by the location of the fracture site. Despite the contrary indications of Figure 6, the failure site was most frequently located (as in Figure 8) away from the apex of the bend and toward the restraining collar (Figure 1). Obviously, this is precisely the location posing the greatest uncertainty in the appropriate choice of analytical formalism, since it represents the transition region between the straight and bent sections of the tube.

Note also that this behavior nicely explains the need for restraining collars on the straight sections of the bent tube to forestall failure there and promote failure in the bent section. Since deformation in the bent section occurs in the region of the tube where the wall thickness is unchanged from that of the as-received tube, and since the tube geometry is unchanged by bending, the only factor dictating the deformation site is the wall strength (Eq. [1]). Obviously, the weaker, undeformed wall in the as-received tube deforms at a lower stress and, hence, lower pressure than the stronger prestrained wall in the bent tube, and, hence, deformation would naturally initiate in the straight, undeformed tube section. Once initiated, deformation continues there because deformation leads to thinning and an increase in tube diameter. These geometric effects ensure that the pressure increase required to sustain deformation in this location is relatively small, so that the pressure required to initiate deformation in the bent section is never attained.

\section{ANALYSIS}

The observed results can be interpreted using a simple mechanics-of-materials approach.

As shown in basic texts, e.g., Reference 7, the circumferential stress $(\sigma)$ in an element of a thin-walled shell with wall thickness of $t_{0}$, under an internal pressure of $P$, can be computed by conducting a force balance on the element. For the special case of a tube* of radius $r$, the result is

*As noted previously, it is arguable that the bent-tube configuration studied in these experiments is more properly analyzed as a section of a torus. The analysis for a torus is developed in the Appendix and demonstrates, for the geometry of interest here, that the results are virtually indistinguishable-the forms of the equations for the straight tube and torus are identical, although there is a minor (4 pct) difference in the numerical factor.

$$
\sigma=\operatorname{Pr} / t_{0}
$$

Clearly, for an isotropic tube of constant wall thickness, the pressure required to induce plastic flow is the same at all locations. However, in a bent tube, the wall thickness and material strength are modified by the strains and associated work hardening resulting from the bending operation. Thus, the pressure required to induce plastic flow will be a function of angular position around the tube.

In a simple bend, assuming isotropic hardening, the flow stresses on two opposing walls will be the same, since both will have experienced the same strains (albeit of opposite sign) and, hence, will have work hardened to the same degree. In the case of interest here, it was noted that stretching was superimposed on the bend. Thus, the total measured strain $(\varepsilon)$ is given by

$$
\varepsilon=\varepsilon_{\text {Bend }}+\varepsilon_{\text {Tensile }}
$$

Clearly, if both $\varepsilon_{\text {Bend }}$ and $\varepsilon_{\text {Tensile }}$ have the same sign, as they do on one side of the tube, the overall work hardening associated with this strain combination is their sum. The situation is a little more complex on the compressive side of the bend, where these two strain contributions are of opposite sign, since some strain relaxation may result.

However, the approach followed in comparing the flow curves of the bent and straight (as-received) tubes - assuming that any relaxation was minimal, so that Bauschinger effects could be ignored-rendered compatible mechanical behavior for these two situations. Thus, a more general, though similar, approach will be followed in analyzing the local mechanical behavior in the bent tube.

First, decompose the measured (true) strain into a bending contribution for all three strain components (tensile, circumferential, and thickness), inferring the axial tensile contribution (previously taken as 5 pct engineering strain) from the measured average axial strain and assuming isotropy to infer the circumferential and thickness components.

Thus, there are three equations.

$$
\varepsilon_{X}^{\text {Measured }}=\varepsilon_{X}^{\text {Bending }}+\varepsilon_{X}^{\text {Tensile }}
$$


Where $X$ refers to the axial, circumferential, and thickness strains, respectively.

These components of the bending and tensile contributions can now be used to determine the effective strains resulting from these deformation modes using the standard formalism. In both cases, the positive root is selected. from

Then, the total deformation experienced can be determined

$$
\varepsilon_{\text {Effective }}^{\text {Total }}=\varepsilon_{\text {Effective }}^{\text {Bending }}+\varepsilon_{\text {Effective }}^{\text {Tensile }}
$$

It is, thus, straightforward to explicitly calculate the expected behavior of the bent tube as a function of orientation by using the measured engineering strains shown in Figure 3 and the observed mechanical behavior of the tube shown in Figure 11, since the measured (true) strains in the bent tube may be modeled as

$$
\varepsilon_{\text {Axial }}^{\text {Measured }}=\ln (1+0.05+0.25 \cos \theta)
$$

and

$$
\varepsilon_{\text {Circumferential }}^{\text {Measured }}=\ln (1+0.02-0.05 \cos \theta)
$$

Thus, the thickness strain may be calculated from

$$
\varepsilon_{\text {Thickness }}^{\text {Measured }}=-\left(\varepsilon_{\text {Axial }}+\varepsilon_{\text {Circumferential }}\right)
$$

and the wall thickness, which obviously varies with angular position, can be calculated from

$$
t=t_{0} \exp \left(\varepsilon_{\text {Thickness }}\right)
$$

Since we are concerned only with the plastic response of the bent tube after appreciable plastic strain, we fit the plastic response of the tube (Figure 11) to the Holloman relation. ${ }^{[8]}$ The best-fit relationship obtained is

$$
\sigma_{\text {Effective }}=865 \cdot \varepsilon_{\text {Effective }}^{0.17} \mathrm{MPa}
$$

Thus, knowing the equivalent strain, we can compute the (equivalent) flow stress in the wall at any location.

Finally, we compute the pressure required for deformation at any angular location in the tube by multiplying the local flow stress by the local thickness and dividing by the tube radius, according to Eq. [1].

Figures 12(a) and (b) show the computed flow stress of the tube wall and the computed deformation pressure after implementing this procedure, respectively.

These computed results are consistent with the observations reported earlier-that the tube, upon pressurization, exhibits only local deformation in the vicinity of the neutral axis and that strain localization is observed on both neutral axes, not simply the one where the tube eventually burst.

Finally, it is worth mentioning that this analysis indicates that the pressure necessary to initiate plastic deformation in the as-bent tube is $\sim 30.5 \mathrm{MPa}-\mathrm{a}$ result which compares quite favorably with the range of 25 to $30 \mathrm{MPa}$ observed in Figures 7 and 9, suggesting that these calculations have been performed in a self-consistent manner.

\section{PRACTICAL IMPLICATIONS}

Unlike free expansion, where geometric effects lead to localized deformation along the length of the tube, the tube-die contact inherent in production hydroforming operations leads to

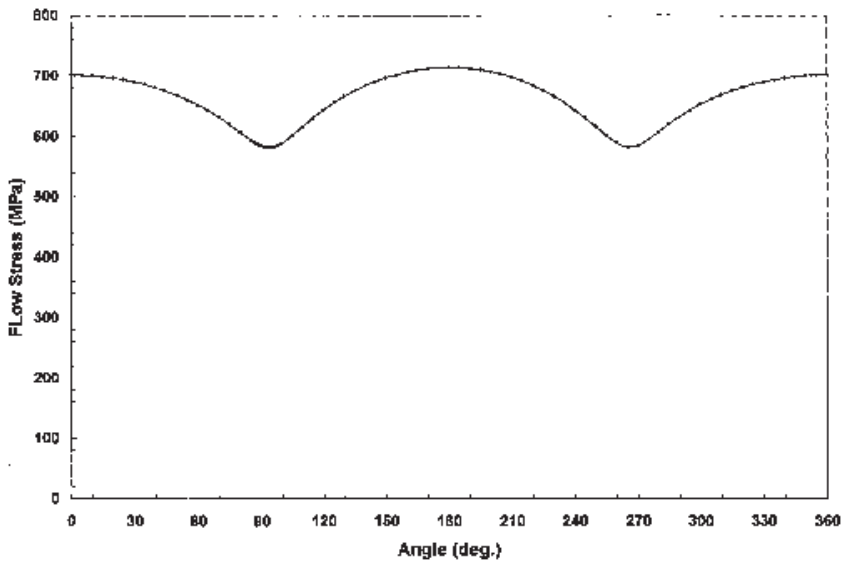

(a)

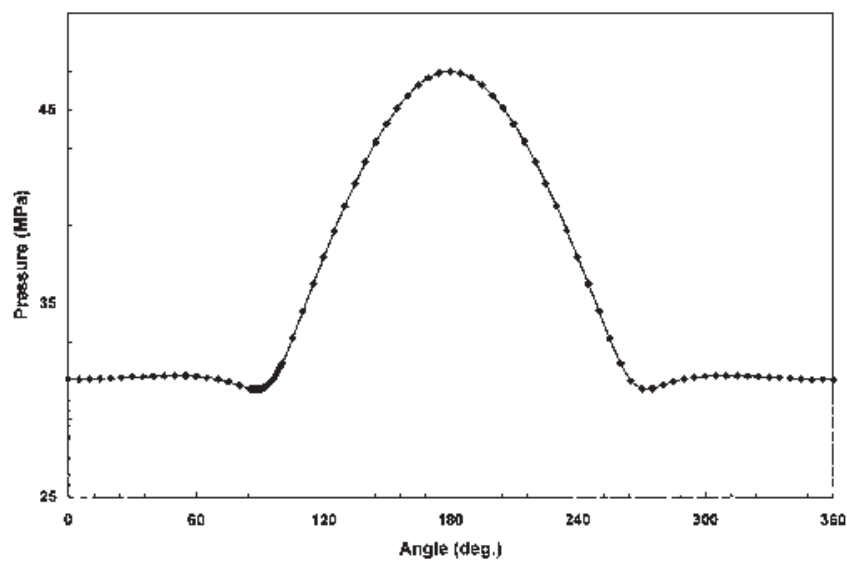

(b)

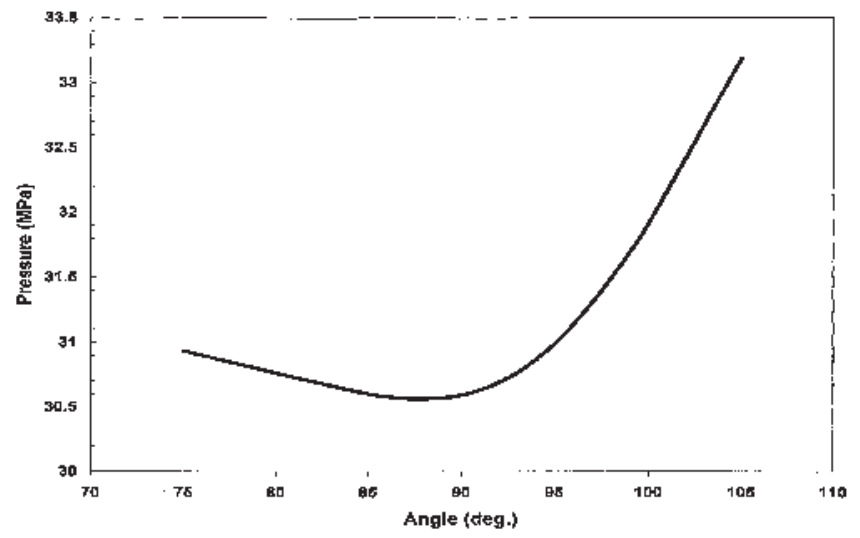

(c)

Fig. 12 - Computed values of $(a)$ wall flow stress (MPa), $(b)$ pressure required to induce deformation in the wall $(\mathrm{MPa})$, and $(c)$ detail of pressure required to induce deformation in the wall (MPa) showing minimum pressure region of Fig. (b) in greater detail, all as a function of angular location in the wall.

much more homogeneous deformation along the length of the tube. Thus, deformation in the bent-tube sections is all but assured. In the event of failure in the bent sections, the conventional approach to analyzing this failure by analyzing the apparent strain history using circle-grid patterns applied to the initially straight tube can lead to apparently anomalous results. These arise because the circle grids are capable of accurately representing the deformation history of the tube wall only when 
no strain-path reversals occur. As illustrated in this study, during imposed bending, some regions of compressive deformation must arise, and if a superimposed tensile stress is applied, then a complex strain path which is not comprehended by a simple comparison of the initial and final circle geometries will result. Thus, depending on where on the bend the failure occurs-the specifics of the deformation process will be driven by the constraints imposed by the die wall and, thus, the deformation may not initiate in the region of lowest flow stress- the failure-strain state recorded by the circle grids may lie comfortably in the "safe" zone in the forming-limit diagram, leading to the erroneous conclusion that the sheet forming-limit diagram is not applicable to hydroforming.

\section{CONCLUSIONS}

1. The deformation of prebent welded tubes under internal pressure is inhomogeneous and is driven by the inhomogeneous strain resulting from bending. The property inhomogeneity associated with the weld has minimal effect.

2. The material response and failure strain associated with internal pressurization are consistent with those which would be expected, provided appropriate account is taken of the prior strain history at the failure site.

3. For the geometry evaluated, equally good agreement with experiment is achieved by analyzing the bent tube as straight or as a section of a torus.

4. Circle-grid strain measurements may be inadequate to capture the complete strain history experienced by the failure element and, thus, may lead to apparent anomalies in the material response, suggesting, erroneously, that conventional forming-limit diagrams are inapplicable to hydroforming.

\section{ACKNOWLEDGMENTS}

All the experiments reported here were carried out at the General Motors Metal Fabricating Division Major Tooling Laboratory. We thank all staff members at that facility for their help and support in conducting this study with particular thanks to Mark Cline, Mark Donoghue, Terry Kent, Ronald Lamarand, Wayne Herring, Ken Johnson, and Mark Hull. We also acknowledge helpful discussions with Michael Wenner, Thomas Stoughton, and Kuo-Kuang Chen.

\section{APPENDIX}

For a torus with geometry given by Figure A1 and subject to an internal pressure $p$, the tangential force $(F)$ in the tube wall at the location specified by $r_{0}$ is given by [A1]:

$$
F=p a\left(r_{0}+b\right) / 2 r_{0}
$$

leading to

$$
\sigma=p a\left(r_{0}+b\right) / 2 r_{0} t_{0}
$$

Noting that failure occurred on the interior of the bend and along the "neutral axis," the region of interest, as shown in Figure 12, is that for which

$$
r_{0}=b-0.27 a
$$

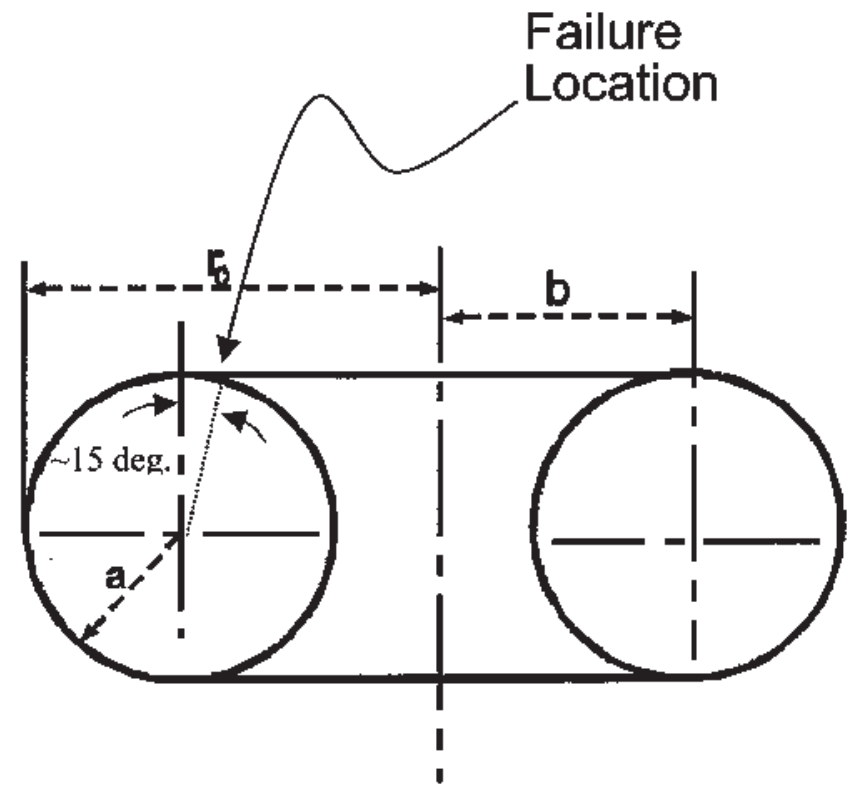

Fig. A1-Geometry and labels for analysis of toroidal pressure vessel ${ }^{[\mathrm{A} 1]}$ showing failure location observed in this study.

Then, in the notation used previously,

$$
\begin{aligned}
\sigma= & P D\left((R-0.14 D+R) / 4 t_{0}(R-0.14 D)\right. \\
= & P D(2 R-0.14 D) / 4 t_{0}(R-0.14 D)
\end{aligned}
$$

where $\mathrm{R}=$ the radius of curvature.

For the case under consideration, a 2-D bend, this simplifies to

$$
\sigma=P D / 2 t_{0} \cdot(1.04)
$$

or, 104 pct of the value for the straight tube (Eq. [1]). Thus, for this geometry, analysis of the results in terms of Eq. [1] given previously is justified, since the forms of the equations are identical, differing only in the numerical factor.

A1. S. Timoshenko and S. Woinowsky-Krieger: Theory of plates and Shells, McGraw-Hill Inc., New York, NY, 1959.

\section{REFERENCES}

1. J. Gerlach, K.W. Blumel, U. Kneiphoff, and G. Eyl: "Material Aspects of Tube-Hydroforming," SAE Technical Paper 1999-01-3204, SAE, Warrendale, PA, 1999.

2. T.B. Stoughton: Int. J. Mech. Sci., 2000, vol. 42, pp. 1-27.

3. A.K. Ghosh and J.V. Laukonis: 9th Biennial Congr. of IDDRG, ASM, Metals Park, OH, 1976.

4. A.F. Graf and W.F. Hosford: Metall. Trans. A, 1993, vol. 24A, pp. 2497-2501.

5. R. Davies, G. Grant, D. Herling, M. Smith, R. Evert, S. Nykerk, and J. Shoup: "Formability Investigation of Aluminum Extrusions under Hydroforming Conditions," SAE Technical Paper 2000-01-2675, SAE, Warrendale, PA, 2000.

6. S. Takahashi, K. Terada, T. Morita, and N. Matsubara: "Application of Hydroforming Simulation on Development of Automobile Parts," SAE Technical Paper 2002-01-0786, SAE, Warrendale, PA, 2002.

7. A. Higdon, E.H. Ohlsen, W.B. Stiles, J.A. Weese, and W.F. Riley: Mechanics of Materials, 3rd ed., John Wiley \& Sons, New York, NY, 1976.

8. J.H. Holloman: Trans. AIME, 1945, vol. 162, p. 268.

9. H. Wang, P. Martin, E. Houghland, F. Feng, and K. Gong: Automotive Tube Hydroforming Conf., Tube and Pipe Association International, Southfield, MI, May 15-16, 2002. 